\title{
LUX - A Recirculating Linac-based Ultrafast X-ray Source
}

\author{
J. N. Corlett, W. A. Barletta, S. DeSantis, L. Doolittle, W. M. Fawley, M.A. Green, \\ P. Heimann, S. R. Leone, S. Lidia, D. Li, F. Parmigiani", A. Ratti, K. Robinson, R. \\ Schoenlein, J. Staples, W. Wan, R. Wells, R. Wilcox, A.Wolski, and A. Zholents
}

Lawrence Berkeley National Laboratory, Berkeley, CA, U.S.A.

* Laboratorio TASC-INFM, Italy

\begin{abstract}
We describe the design of a proposed source of ultra-fast synchrotron radiation x-ray pulses based on a recirculating superconducting linac, with an integrated array of ultrafast laser systems. The source produces x-ray pulses with duration of 10-50 fs at a $10 \mathrm{kHz}$ repetition rate, with tunability from EUV to hard x-ray regimes, and optimized for the study of ultra-fast dynamics. A high-brightness rf photocathode provides electron bunches. An injector linac accelerates the beam to the $100 \mathrm{MeV}$ range, and is followed by four passes through a $700 \mathrm{MeV}$ recirculating linac. Ultrafast hard x-ray pulses are obtained by a combination of electron bunch manipulation, transverse temporal correlation of the electrons, and x-ray pulse compression. EUV and soft x-ray pulses as short as $10 \mathrm{fs}$ are generated in a harmoniccascade free electron laser scheme. We describe the facility major systems and peformance
\end{abstract}

\section{OVERVIEW}

LUX is a proposed facility to produce ultrashort x-ray pulses in a highly refined manner for experiments across all fields of the physical, chemical and biological sciences [1,2]. Based on a recirculating electron linac, the LUX proposal is a compact and cost-effective configuration for production of intense ultrafast EUV and x-ray probe pulses, with tight synchronization to a range of femtosecond pump laser systems. Providing a broad photon spectrum covering the whole range from EUV to hard x-ray allows both spectroscopy and diffraction capabilities, probing both nuclear positions and electronic, chemical or structural properties. The design $10 \mathrm{kHz}$ pulse repetition rate is matched to pump-probe experiments and allows rapid data acquisition, and sample relaxation or complete sample regeneration by translation or flow.

A recirculating linac accelerates 2 ps electron bunches to $\sim 3 \mathrm{GeV}$, where the electrons radiate in a selection of insertion devices. Intense soft $\mathrm{x}$-rays are produced by harmonic-cascade FEL scheme, similar to high-gain harmonic-generation (HGHG) - a laser-seeded process in a cascaded series of undulators, resulting in enhanced radiation at selected harmonics of the seed $[3,4]$. The coherent soft $\mathrm{x}$-rays can be tuned by adjusting seed laser wavelength and undulator settings, simulations indicate that a range of tens of $\mathrm{eV}$ to $\sim 1 \mathrm{keV}$ is achievable, with ultrashort seed laser pulses producing x-ray pulse durations of 10-200 fs. Hard x-rays are produced by spontaneous emission of the electrons in narrow-gap, short-period undulators. By use of a novel bunch tilting process followed by optical compression, hard x-ray pulse durations of 50-100 fs are obtained over a range of 1-10 keV [1,5].

Synchronization of the x-rays with sample excitation lasers is critical for time-domain experiments, and optical pulses are distributed from a laser master oscillator to provide timing signals and conversion to rf signals around the facility. These pulses are used to synchronize harmonic-cascade seed lasers, experimental end station lasers, and accelerator laser and rf systems, by generation of rf and microwave signals in fast photodiodes, followed by phaselocked loops operating on each laser system. The processes used to generate x-rays in LUX are inherently insensitive to timing jitter in the electron bunches, and synchronization at the level 10-50 fs is expected.

The proposed LUX facility would provide stable, synchronized, tunable, ultrafast $\mathrm{x}$-ray pulses with variable polarization, and with peak fluxes comparable to third generation light sources, to multiple beamlines operating simultaneously over a broad range of x-ray wavelengths. An integrated array of lasers at the beamline endstations allows flexibility in sample excitation. 


\section{ACCELERATOR}

Electron pulses are produced at a rate of $10 \mathrm{kHz}$ in high-brightness rf photocathode guns. Two interleaved sources are used - one with a conventional circular cross-section beam optimized for production of high-brightness EUV and soft x-ray radiation in the harmonic-cascade scheme, the other with a large $\mathrm{x} / \mathrm{y}$ emittance ratio or "flat" beam for production of hard x-rays. The beam quality requirements of the rf photocathode guns are already demonstrated, with normalized emittance of approximately $3 \mathrm{~mm}$-mrad at $1 \mathrm{nC}$ charge, although the high repetition rate has not been addressed to date. For the LUX facility we have produced a conceptual design optimized for operation at high gradient and high repetition rate, and producing low-emittance bunches. This design incorporates features that increase cavity surface area to reduce deposited power density and enhance the accelerating electric field at the cathode $\left(82 \mathrm{~W} / \mathrm{cm}^{2}\right.$ maximum power density for $64 \mathrm{MV} / \mathrm{m}$ at the cathode) [1].

A beam with significantly reduced emittance in one plane is produced by application of a solenoidal magnetic field on the cathode, followed by a specially configured skew-quadrupole channel. The emittances of the left/right circular modes in the magnetized beam are converted to the $\mathrm{x} / \mathrm{y}$ planes following the skew-quadrupole channel, allowing production of an $\mathrm{x} / \mathrm{y}$ emittance ratio 50/1 and small vertical normalized emittance of $0.4 \mathrm{~mm}$-mrad. This technique has been successfully demonstrated with properties close to LUX design parameters [6]. The small emittance is important in producing ultrafast hard x-ray pulses [1].

In the transport line from the $100 \mathrm{MeV}$ injector linac to main linac the bunches are compressed from $\sim 20$ ps to 2 ps. This transport line is carefully designed to compensate for the effects of coherent synchrotron radiation at the shorter bunch lengths.

In the recirculating linac the maximum energy of $\sim 3 \mathrm{GeV}$ is achieved after four passes through the $700 \mathrm{MeV}$ superconducting rf structure. The electron bunch repetition interval in LUX is less than the superconducting cavity filling time, and the linacs are more efficiently operated in $\mathrm{cw}$ mode. Engineering modifications to existing cryomodule designs required to accommodate significantly increased thermal load in the liquid helium are described in [1]. Significant advances have been made in superconducting $\mathrm{rf}$ technology in recent years, and the parameters of the TESLA superconducting rf systems developed at DESY have been used in LUX design studies, and design concepts from Jefferson Lab meet the needs for LUX [7,8].

The flexibility of the LUX lattice allows control and preservation of electron beam transverse and longitudinal emittances, minimizing the influence of collective effects. The lattice is designed to allow manipulation of the bunch phase space on each pass if required, and also to accommodate bunch rates greater than the $10 \mathrm{kHz}$ baseline design.

\section{PHOTON PRODUCTION}

At the exit of the final arc the flat-beam electron bunches receive a time-correlated vertical kick in a deflecting rf cavity. This imparts to the electron bunch a transverse momentum that is correlated in amplitude to longitudinal position within the bunch. The electrons then radiate x-rays in the downstream chain of undulators and wiggler magnets, imprinting this correlation in the geometrical distribution of the x-ray pulse. The correlated x-ray pulse is then compressed to 10's fs duration by use of asymmetrically cut crystal optics.

There are several qualitative differences between LUX and electron storage ring requirements for hard x-ray optics and beamlines. Firstly, the beamline must accommodate the position or angle correlation of the electron bunch - in the wigglers, electrons will have a vertical position-time correlation, in the undulators, a vertical angletime correlation. Secondly, the average current is low, $10 \mu \mathrm{A}$, and consequently, the total power radiated by the undulators and wigglers is also low, typically $0.4 \mathrm{~W}$. None of the high power optical engineering typical of the third generation synchrotron radiation sources is required, and silicon or fused silica optics can be used without water cooling. The design of the photon stops in the front ends is also simplified.

Narrow-gap in-vacuo superconducting undulator designs provide tunable high-flux sources of spontaneous emission hard x-rays. A typical undulator will have $2 \mathrm{~m}$ length, $1.5 \mathrm{~cm} \mathrm{p} \quad, 4 \mathrm{~mm}$ gap and $2 \mathrm{~T}$ peak field on axis, resulting in an optimal photon energy range of 1.4 to $12 \mathrm{keV}$ at $3 \mathrm{GeV}$ beam energy. The spectral shape of the undulator harmonics is modified by the angular correlation of the electron bunches, and the undulator beamline vertical acceptance is increased from 50 to $500 \mu \mathrm{rad}$ in order to accept the larger angular divergence of the beam. The angular correlation broadens the undulator harmonic, but the peak flux is unchanged. This result can be understood from the fact that each electron is radiating essentially the same spectrum but with a variation of the onaxis direction. The brightness is, however, decreased by the increase in the angular divergence. 
Wigglers provide intense hard x-ray sources with a continuous spectrum extending to high photon energy. However, because wigglers produce incoherent radiation, the depth of source effect must be considered. The hard $\mathrm{x}$-ray pulse compression scheme depends on the small vertical source size. The effective source is calculated by projecting the radiation from along the length of the wiggler onto a vertical plane at the center and then convoluting with the electron beam [9]. Considering the case of a $60 \mathrm{~cm}$ long wiggler and $0.2 \mathrm{mrad}$ vertical acceptance, the effective source size is $33 \mu \mathrm{m}$ (fwhm) compared with $26 \mu \mathrm{m}$ (fwhm) for the electron beam. Consequently, wigglers can be used at LUX consistent with the x-ray pulse compression scheme.

Asymmetrically cut crystals may be used as optical elements in a hard x-ray pulse compression scheme [5]. As a result of the different angles of incidence and diffraction, a crystal may be oriented to produce a variable path length across the $\mathrm{x}$-ray beam. When the variation in the optical path length matches the spatial correlation of the incident $\mathrm{x}$ rays an ultrafast $\mathrm{x}$-ray pulse is produced. Two crystals are used in order to provide a fixed exit beam direction while changing the photon energy.

A laser-seeded harmonic-cascade FEL scheme produces high-flux, short-pulse photons over an energy range of tens of eV to $\sim 1 \mathrm{keV}$. In this process the circular cross-section high-brightness electron beam is extracted from the recirculating linac, and passed through an undulator where a co-propagating seed laser modulates the charge distribution over a short length of the bunch. The harmonic-generation scheme has been developed and demonstrated at the Brookhaven DUV FEL facility [4]. The imposed modulation results in enhanced radiation at specific wavelengths and a selected wavelength is amplified in a following undulator, tuned to a higher harmonic of the seed laser. The electron pulse is then delayed in a short chicane, and the process repeated by modulating a fresh portion of the beam this time with the harmonic radiation produced in the previous undulator. Using an optical parametric amplifier as the seed and variable undulators allows significant tunability in four stages of harmonic generation, flux up to $10^{13}$ photons per pulse, and variable pulse duration depending on the seed laser parameters [3]. Two chains of cascaded harmonic generation are proposed, providing exceptional flexibility in producing EUV and soft $\mathrm{x}$-ray pulses. Circular polarization may be attainable by use of elliptical undulators, and flux stability of $0.1 \%$ or better is obtained in seconds from random pulse-pulse flux variations of $10-20 \%$ at $10 \mathrm{kHz}$ repetition-rate. The use of tapered undulators would allow tailoring of flux to individual experiments.

As a further development, schemes to produce attosecond soft x-ray pulses are being pursued. Using high-power lasers only a few cycles in duration and the flexibility of the seeded FEL approach may allow an additional reduction in pulse duration by perhaps two orders of magnitude, to the 100 attosecond regime.

\section{LASERS AND SYNCHRONIZATION}

Sophisticated laser systems will be an integral part of the LUX facility, providing experiment excitation pulses, and stable timing signals, as well as the electron source through the photocathode laser. Each endstation will have it's own dedicated laser system and optical manipulation and diagnostics, and optical tables and equipment will be contained within a stable and controlled environment. Multiple tuneable lasers covering a range of 267-3000 nm and pulse durations of $\leq 50$ fs are required for experiment initiation, together with temporal and spatial filtering to optimize performance for specific experimental applications.

Distribution systems using fiber-optic transmission lines will provide optical seed pulses from the laser master oscillator to each beamline, with path lengths stabilized by feedback based on optical interferometric measurements. Developments in laser technology are expected to result in significant improvements in the coming years, which will be incorporated into our design with minimal impact on accelerator systems.

Synchronization of the ultra-short $\mathrm{x}$-ray pulses to the experimental excitation pulse is critical to studies of ultrafast dynamics. For LUX we propose to generate inherently stable pulses by using seeded FEL systems, and bunch manipulation followed by x-ray compression. In the case of EUV and soft x-ray production, the cascaded harmonic generation seed laser oscillator also drives the sample excitation laser, resulting in timing stability of approximately 20 fs. For our scheme of hard x-ray production by bunch manipulation followed by x-ray pulse compression, we find that the phase jitter of the deflecting cavities with respect to the experimental laser pulse dominates timing issues [1]. Phase and amplitude feedback of the deflecting cavities is expected to provide x-ray pulse to laser pulse timing stability of $50 \mathrm{fs}$ or better. To stabilize all timing and rf signals in the facility, we propose to use a phaselocked laser oscillator as the facility master oscillator. The rf gun, linacs, and deflecting cavities may thus be phaselocked to the experimental excitation lasers, and timing jitter between the optical laser and the x-ray pulse emitted by the beam minimized. 


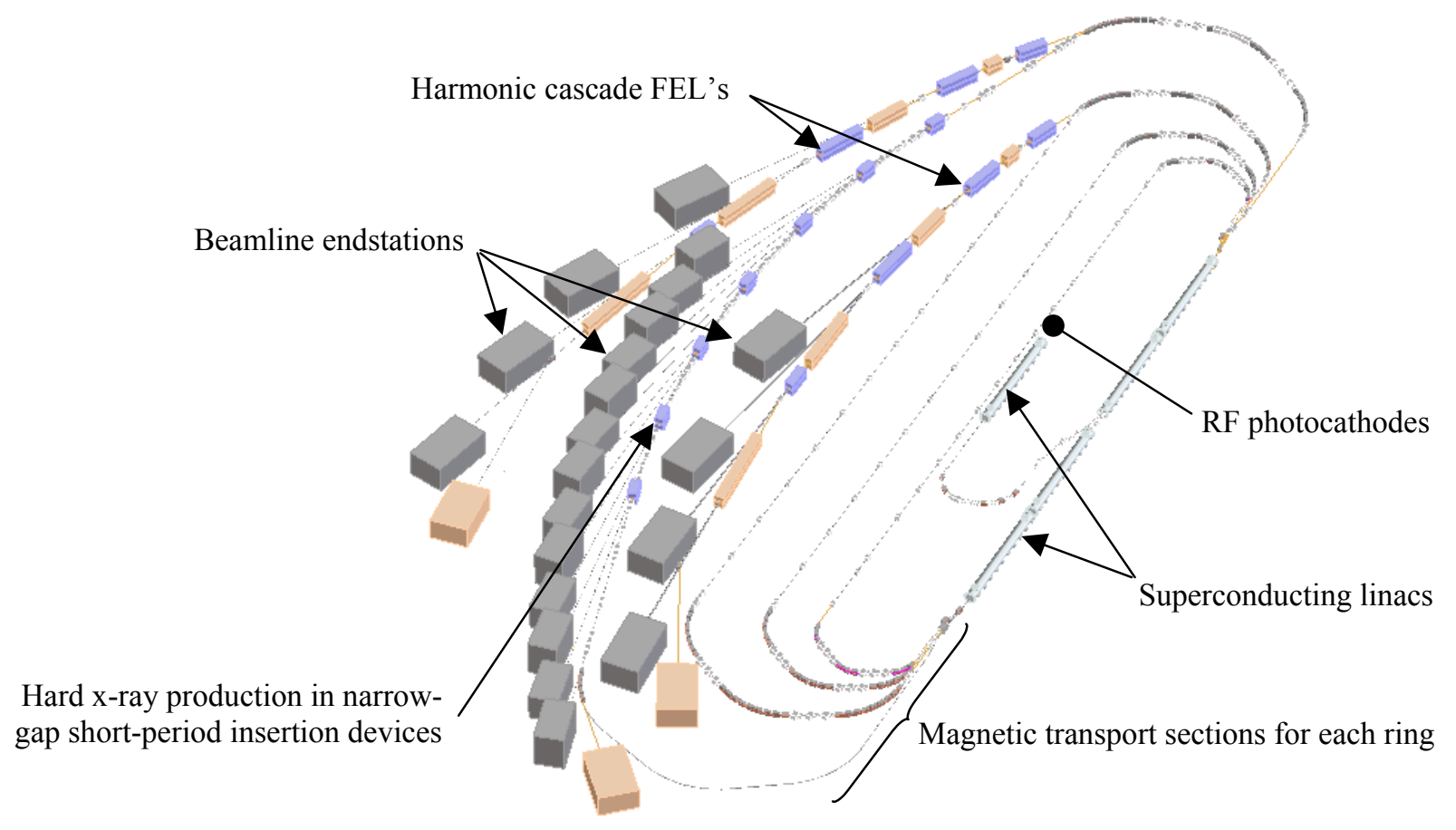

FIGURE 1. Machine layout. The beams generated at the rf photocathode guns travel through the injector linac, main linac, transport arcs, and either hard x-ray production section or a cascaded harmonic generation section, to the beam dump.

\section{SUMMARY}

LUX is a recirculating linac-based facility proposed to address the growing national and international need for ultrafast X-ray scientific research. The facility is being specifically designed with a view toward solving problems in ultrafast science, with integrated laser systems, tunable x-ray beamlines covering a wide spectrum, and tight synchronization between laser pump and $\mathrm{x}$-ray probe beams.

\section{ACKNOWLEDGEMENTS}

This work was supported by the U.S.Department of Energy under Contract No. DE-AC03-76SF00098

\section{REFERENCES}

1. J. N. Corlett, W. Barry, W. A. Barletta, et al, "Feasibility study for a recirculating linac-based facility for femtosecond dynamics", LBNL formal report LBNL-51766, December 2002.

2. J. N. Corlett, W.A. Barletta, S. De Santis, et al "A Recirculating Linac-Based Facility for Ultrafast X-ray Science", Proc. 2003 Particle Accelerator Conference, Portland, Oregon, May 12 - 16, 2003, May 2003.

3. W. Fawley et al, "Simulation studies of an XUV/soft x-ray harmonic-cascade FEL for the proposed LBNL recirculating linac", Proc. 2003 Particle Accelerator Conference, Portland, Oregon, May 12 - 16, 2003, May 2003.

4. L.-H. Yu et al, "High-Gain Harmonic-Generation Free-Electron Laser", Science 289 932-934 (2000).

5. A. Zholents et al "Generation of subpicosecond x-ray pulses using RF orbit deflection", NIM A 425 (1999) 385-389, 1999.

6. D. Edwards et al, "The Flat Beam Experiment at the FNAL Photoinjector", Proc. XXth International Linac Conference, Monterey, 2000.

7. TESLA Technical Design Report, DESY 2001-011, March 2001.

8. L. Harwood, C. Reece, "CEBAF at 12 and 25 GeV", Proc. SRF2001, Tsukuba, Japan, Sept. 2001.

9. R. Coisson and R.P. Walker, "Phase space distribution of brilliance of undulator sources," SPIE Proceedings 582, pp.24-29, 1986. 